\title{
Notes anatomiques sur l'ovaire de quelques Papavéracées
}

\author{
(Suite et fin) ${ }^{4}$; \\ PAR M. 0. LIGNIER. \\ Eschscholtziées.
}

D'après Prantl et Kündig ${ }^{2}$ chacun des deux carpelles qui forment l'ovaire des Dendromecon, se termine par un stigmate trilobé et chacun des lobes stigmatiques latéraux est desservi par les faisceaux placentaires correspondants ${ }^{3}$.

Chez l'Eschscholtzia californica Cham. l'ovaire bicarpellé se termine habituellement par quatre longs stigmates cylindriques
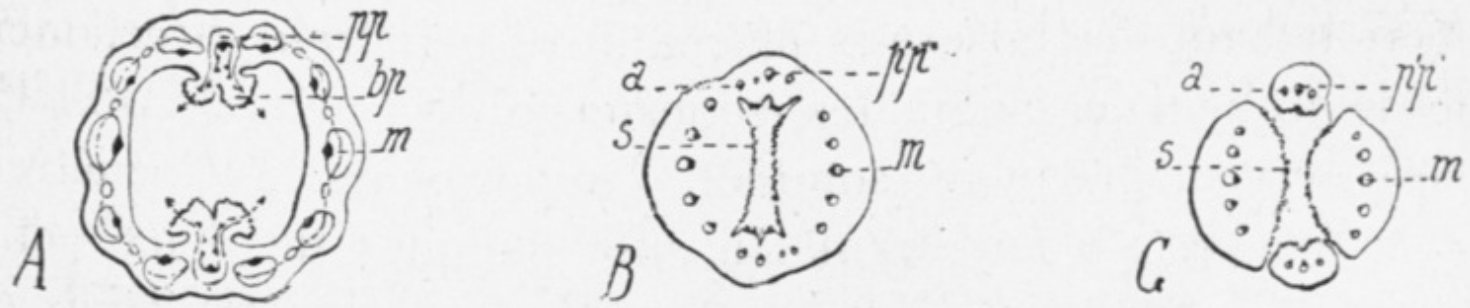

Fig. 9. - Sections transversales de l'ovaire de l'E. californica : A, au milieu de l'ovaire; B, au niveau du style; C, dans la base des stigmates, Gr. 8/2. - $m$, faisceaux carpellaires médians; $p p$, faisceaux placentaires; $p^{\prime} p^{\prime}$, faisceaux placentaires prolongés dans les stigmates; $b p$, bourrelets placentaires; $a$, faisceaux descendants dans les bords du limbe stérile des carpelles; $s$, surface stigmatique glandulaire.

dont les deux plus grands prolongent le milieu des carpelles et dont les plus petits, en croix avec les précédents, sont situés au-dessus des placentas. Mais il peut parfois exister des stigmates supplémentaires qui, d'après Baillon, sont alors plus courts que les précédents et se montrent comme des dépendances latérales des deux grands médians ${ }^{4}$. D'autre fois, dans les fleurs grêles, on n'en observe que deux et ce sont toujours ceux qui prolongent le milieu des carpelles.

Les placentas de l'E. californica sont de la forme en bour-

1. Voir plus haut, p. 279 et p. 337.

2. Pflanzenfamilien, t. III, 2 Ab, p. 13 -138 (fig. $86, \mathrm{C}$ ).

3. Je n'ai pu, par manque d'échantillons, vérifier cette affirmation qui d'ailleurs semble être parfaitement justifiée.

4. Voir BallLon, Histoire des Plantes, t. III. p. 119, et PAYER, Organogén ie végétale (pl. XLV, fig. 38). 
relets accouplés, A (fig. 9), déjà reconnue chez les Chelidonium. Ils sont bien saillants et portent de nombreux ovules. Un seul faisceau longitudinal en fer à cheval, plus ou moins lobé, $p p$ (fig. 1, A), les dessert-dans toute leur longueur; il a ses bords recourbés vers les bourrelets placentaires et $y$ donne insertion à tous les cordons ovulaires. Par places ce faisceau placentaire, en apparence indépendant du limbe stérile des carpelles, se rattache cependant à son réseau par des commissures transversales.

Ainsi done l'organisation des placentas dans l'ovaire de l'Eschscholtzia californica offre une grande analogie avec ce que j’ai précédemment décrit chez les Chelidonium et même, a part l'importance placentaire et la multilobation du faisceau placentaire, avec ce que montre le Glaucium flavum; elle est, en quelque sorte, intermédiaire entre les deux.

Vers le haut, les faisceaux placentaires pénètrent directement dans le milieu des stigmates superposés, $p^{\prime} p^{\prime}$ (fig. 9, B et C), et les suivent jusqu'au sommet. Toutefois ils n'y pénètrent pas seuls; ils s'y montrent en effet flanqués de deux petits faisceaux a qui, vers le bas, sont en rapport avec les bords du limbe stérile des carpelles.

Ajoutons encore que la face intérieure de ces petits stigmates est, au moins sur leur base, creusé d'un sillon, prolongement de celui qui se trouve entre les bourrelets ovulifères.

Dans les grands stigmates pénètre tout le système libéroligneux de la partie stérile des limbes carpellaires, moins les petits faisceaux latéraux $a$, qui, ainsi que je viens de le montrer, se rendent aux bords des petits stigmates. Il est vraisembable que lorsque les grands stigmates médians sont lobés comme l'indique Baillon, l'ensemble de leur système libéro-ligneux se repartit entre les lobes.

Le tissu de déhiscence des valves a, aux niveaux ovulifères, la īième position que chez les Chélidoniées, mais, vers le haut, il se poursuit verticalement de manière à détacher les grands stigmates des petits.

Le cas de l'Eschscholtzia est tout particulièrement intéressant parce que, pour qui le considérerait séparément, il pourrait offrir de magnifiques arguments à l'appui de l'opinion d'après 
laquelle l'ovaire des Papavéracés comprend deux verticilles alternes de carpelles les uns stériles les autres fertiles. C'est du reste en effet sur ce genre que s'est surtout appuyé Lindley pour défendre cette interprétation.

Mais la comparaison de la structure ovarienne avec celles du Chelidonium et du Gl. flavum permet d'éviter une telle erreur d'interprétation et de comprendre la vraie signification des faits observés.

En réalité, de mème que celui des Platystémonées et des Chélidoniées, l'ovaire de l'Eschscholtzia ne renferme qu'un seul verticille carpellaire. Dans sa région ovulifère, je l'ai déjà dit, les placentas diffèrent à peine de ceux du Chelidonium dont ils ont presque la simplicité - ils sont beaucoup moins complexes que ceux du Gl. flavum. - Ce n'est que plus haut que les différences apparaissent. Mais déjà les Chélidoniées nous les avaient laissé prévoir par la connaissance du Gl. flavum. Nous avions en effet constaté que, chez cette dernière espèce, il y avait une sorte de tendance à la trilobation, tendance qui, il est vrai, n'était encore indiquée, au stigmate, que par un élargissement de sa base en languette transversale, mais que nous retrouvons complètement caractérisée chéz le Dendromecon où la trilobation des stigmates est nettement représentée.

Dès lors, guidés par la connaissance de la valeur exacte des régions ovulifères acquise chez les espèces précédentes, et aussi par celle des faits ci-dessus dans les stigmates du Gl. flavum et du Dendromecon, il va nous être facile de comprendre le stigmate multifide de l'Eschscholtziu. Ses gros lobes correspondent aux stigmates médians des carpelles du Dendromecon; ses petits lobes superposés aux placentas, résultent de la coalescence deux à deux des lobes stigmatiques latéraux de ce mème genre. Si chacun d'eux ne renferme qu'un seul faisceau médian au lieu de deux qu'on pourrait s'attendre à y rencontrer, cela provient très certainement de l'énergie de la fusion des lobes en question. Il s'est produit là un fait analogue à celui que nous avons dù constater en comparant les placentas unifasciculés du Macleaya cordata aux placentas bifasciculés du Platystemon et du Meconella.

Je n'ai pas eu la possibilité d'étudier la structure de l'ovaire 
du Hunnemannia, mais, d'après les descriptions qui en sont données, il semble qu'on devrait y trouver, au moins en ce qui concerne les stigmates, une organisation intermédiaire à celle du Dendromecon et de l'Eschscholtzia.

\section{CONCLUSIONS.}

Chez toutes les Papavéracées comprises dans cette étude, l'ovaire ne renferme jamais qu'un seui verticille de carpelles. L'extrémité supérieure de chacun de ces carpelles est transformée en stigmate. Celui-ci est d'ordinaire entier, mais, déjà élargi à la base chez le Glaucium flavum de manière à former des sortes de languettes transversales, il est nettement trilobé chez le Dendromecon et l'Eschscholtzia.

Chaque carpelle est desservi par trois faisceaux principaux que relie un réseau de veinules libéro-ligneuses. De ces trois faisceaux le médian, d'ordinaire le plus petit des trois au moins aux niveaux ovulifères, se poursuit jusque dans le sommet du stigmate; les deux latéraux qui desservent les placentas, se réunissent le plus souvent au précédent près de son extrêmité. Toutefois, chez le Dendromecon et l'Eschscholtzia ils restent isolés mème dans leur parcours supérieur et pénètrent dans les stigmates latéraux.

La coalescence marginale des carpelles est excessivement faible chez le Platystemon et le Meconella; elle n'y intéresse que le tissu parenchymateux de telle sorte que les faisceaux marginaux ou placentaires d'une même région de coalescence restent séparés. A chacun d'eux correspond alors un bourrelet placentaire qui ne porte qu'une seule ligne d'ovules et les cordons vasculaires de ces ovules s'insèrent sur le bord externe (le plus rapproché des marges carpellaires) des faisceaux placentaires. La déhiscence des valves du fruit se fait entre les faisceaux placentaires, à la marge même des carpelles.

Chez tous les autres genres étudiés la coalescence marginale des carpelles est beaucoup plus intime et les faisceaux marginaux eux-mèmes y participent en se fusionnant deux à deux en un faisceau placentaire unique. Celui-ci d'ordinaire représenté par un seul cordon libéro-ligneux à bords plus ou moins incur- 
vés vers l'intérieur, peut parfois, lorsqu'il y a beaucoup d'ovules (Platystigma lineare, Glaucium flavum, parfois Eschscholtzia californica) l'être par tout un plexus de cordons.

En ce qui concerne l'insertion des ovules sur ce faisceau unique ou sur ce plexus deux cas peuvent se rencontrer. Chez le $\mathrm{Pl}$. lineare les ovules sont répartis en grand nombre sur toute la largeur d'une bande placentaire parallèle au plexus et leurs cordons vasculaires s'insèrent indifféremment sur l'un ou l'autre bord (externe ou interne) des faisceaux de ce plexus qui leur font vis-à-vis. Chez tous les autres genres étudiés les ovules sont répartis en deux files et ils s'insèrent les uns sur un des bords, les autres sur l'autre de l'unique faisceau placentaire ou du plexus qui le remplace. Il y a lieu de noter que dans ce cas l'un et l'autre bord de l'unique faisceau placentaire correspondent aux bords internes des faisceaux placentaires séparés du Platystemon et du Meconella, de telle sorte qu'une des conséquences de l'accentuation de la concrescence des carpelles et, par suite, du déplacement de la ligne de concrescence de leurs marges à leurs faisceaux marginaux, est de changer le lieu d'insertion des ovules. Primitivement insérés sur le bord externe des faisceaux placentaires ils reportent finalement leur insertion sur le bord interne de ces mèmes faisceaux.

C'est là un fait qui, à première vue, peut paraître singulier et qui cependant s'explique facilement si l'on se souvient que les différentes parties du mériphyte foliaire sont, dans la feuille, insérées les unes sur les autres de la mème façon que dans la tige, les mériphytes foliaires successifs sont insérés les uns sur les autres ${ }^{1}$. On comprend dès lors en effet, que des variations dans les rapports de position des pièces desservies par Ies parties du mériphyte, suffisent pour provoquer des variations dans les lieux d'insertion de ces parties. Les folioles fertiles (ovules) étant déplacées vers les plans carpellaires par l'accroissement de la concrescence carpellaire, les insertions de ces folioles se trouvent également déplacées dans le mème sens.

D'ordinaire la concrescence libéro-ligneuse des carpelles ne se produit qu'aux niveaux ovulifères ou au plus dans la base du

1. Voir Lignier (0.), De l'influence de la symétrie que la tige exerce sur la distribution, le parcours et les contacts de ses faisceaux libero-ligneux (Bull. Soc. Linn. de Normandie, déc. 1888-avril 1889, Caen). 
style. Plus haut les deux faisceaux constituants de l'unique faisceau placentaire reprennent leur liberté et s'en vont soit progressivement rejoindre le faisceau carpellaire médian au sommet du stigmate, soit sortir séparément dans le lobe latéral correspondant du stigmate si celui-ci est trilobé (Dendromecon). Cependant chez l'Eschscholtzia où les stigmates sont également trilobés la coalescence des carpelles se poursuit jusqu'en haut, fusionnant en un seul dans le plan placentaire les deux lobes latéraux voisins, et cette coalescence $\mathbf{y}$ reste si intime que le faisceau placentaire unique se poursuit jusque dans le sommet de ces lobes stigmatiques intercarpellaires.

La déhiscence des valves, dans les genres à faisceau placentaire unique se prépare souvent de très bonne heure au moyen d'une lame de tissu spécialisé qui coupe de nombreux faisceaux libéro-ligneux, soit seulement les commissures qui unissent les faisceaux placentaires au limbe stérile des carpelles, soit en plus le faisceau médian carpellaire lui-mème. Il peut en résulter pour ces faisceaux un amoindrissement qui semble être en rapport avec la précocité de la différenciation des tissus de rupture.

Chez le Platystigma lineare dont les ovules sont nombreux et plus encore chez le Glaucium flavum dont les nombreux ovules sont tous insérés sur les bords du plexus placentaire, les placentas prennent l'aspect de carpelles fertiles intérieurs, alternant avec les carpelles stériles extérieurs (limbe stérile des carpelles). Cet aspect est encore accentué par le développement du tissu de déhiscence des valves qu'on pourrait prendre pour un tissu de suture, et, en particulier, chez l'E. californica, par la formation d'un plissement longitudinal du limbe carpellaire plissement qui repousse brusquenent les placentas et leurs plexus vers l'intérieur.

\section{Appendice.}

Des recherches encore inachevées m’incitent à admettre que chez les Papavérées et les Fumariées l'ovaire n'est également jamais constitué que par un seul verticille de carpelles. Si parfois il semble en renfermer deux, un extérieur stérile et un intérieur fertile, c'est en raison de faits comparables à ceux décrits chez le Platystigma et le Glaucium. N'en est-il pas de même chez les Crucifères et les Capparidées? 
Chez les Papavérées les apparences sont souvent compliquées par l'organisation des placentas et presque toujours par certaines modifications particulières de l'appareil stigmatique.

C'est ainsi que donnant insertion à de nombreux ovules, les faisceaux placentaires peuvent ètre, comme chez le Glaucium, représentés chacun par un plexus en arc, mais alors celui-ci y est beaucoup plus incurvé et correspond à un placenta qui se prolonge radialement dans la cavité ovarienne; parfois même ce plexus est en couronne, comme chez le Platystigma.

Dans le style ces faisceaux placentaires (ou ces plexus) se dédoublent en leurs composants (carpellaires marginaux), puis ceux-ci se divisent à leur tour, dans la base très élargie des stigmates, chacun en un réseau qui s'étend progressivement de la marge du stigmate jusqu'au plan carpellaire médian. Les deux réseaux latéraux d'un même carpelle se fusionnent ensuite et se terminent dans le sommet du stigmate.

En somme les faits sont dans le sommet des carpelles les mèmes que chez les Chélidoniées et les Eschscholtziées, les trois faisceaux carpellaires principaux se réunissent et se fusionnent dans le sommet $d u$ carpelle, mais, un peu comme chez le Glaucium, chaque faisceau latéral est, dans le stigmate, très élargi et représenté par un réseau. En outre, d'ordinaire, le faisceau médian est plus ou moins atrophié à son extrémité supérieure. Il s'éteint souvent au niveau où il est coupé par le tissu de déhiscence des valves et vraisemblablement sous l'influence de ce tissu. D'autre part, l'élargissement basilaire du stigmate peut ètre accompagné d'une lobation analogue à celle du Dendromecon ou de l'Eschscholtzia. Enfin, les bords simples ou lobés de ce stigmate élargi sont toujours d'autant plus recourbés en arrière que le nombre des carpelles dont se compose l'ovaire, est plus élevé; et ils tendent ainsi à former, surtout s'ils sont très élargis, un appareil stigmatique radié en apparence très compliqué.

'Chez les Crucifères et les Capparidées ce sont vraisemblablement ces marges élargies ou même lobées des stigmates qui, à peu près seules, conservent la fonction glandulaire et donnent l'apparence extérieure de stigmates superposés aux placentas. 


\section{$2 \mathrm{BHL}$ Biodiversity Heritage Library}

Lignier, Octave. 1911. "Notes anatomiques sur l'ovaire de quelques Papavéracées (Suite et fin)." Bulletin de la Société botanique de France 58, 429-435. https://doi.org/10.1080/00378941.1911.10831446.

View This Item Online: https://www.biodiversitylibrary.org/item/8680

DOI: https://doi.org/10.1080/00378941.1911.10831446

Permalink: https://www.biodiversitylibrary.org/partpdf/161021

\section{Holding Institution}

Missouri Botanical Garden, Peter H. Raven Library

\section{Sponsored by}

Missouri Botanical Garden

\section{Copyright \& Reuse}

Copyright Status: Public domain. The BHL considers that this work is no longer under copyright protection.

This document was created from content at the Biodiversity Heritage Library, the world's largest open access digital library for biodiversity literature and archives. Visit BHL at https://www.biodiversitylibrary.org. 